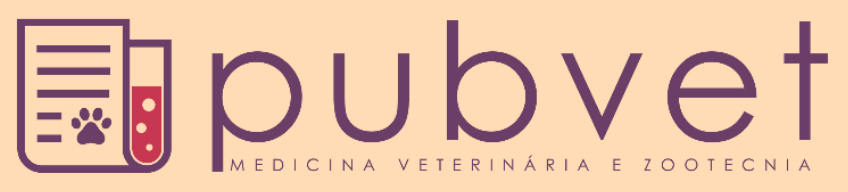

https://doi.org/10.31533/pubvet.v13n5a337.1-7

\title{
Encarceramento intestinal em forame epiplóico: Relato de caso em equino
}

\author{
Verônica Souza Santos ${ }^{1}$, Dilma Mendes de Freitas ${ }^{2 *} \bullet$, Franciele Júlia Coelho Borges ${ }^{1} \bullet$, Laiane Cintra \\ Alves $^{1}$, Ana Luiza Teixeira Amado Jorge ${ }^{30}$, Marcos Figueiredo Pereira ${ }^{4}{ }^{\theta}$,João Victor da Silva e Silva ${ }^{5}$, \\ Maria Eduarda de Lourdes $\operatorname{Vaz}^{50}$, Sabrine Morais dos $\operatorname{Santos}^{2}{ }^{\ominus}$, Cândice Mara Bertonha ${ }^{6}{ }^{\ominus}$
}

\author{
${ }^{I}$ Médica Veterinária, graduada pela Universidade de Uberaba. Uberaba-MG Brasil. \\ ${ }^{2}$ Aprimoranda em Patologia Veterinária no Hospital Veterinário de Uberaba. Uberaba - MG Brasil. \\ ${ }^{3}$ Médica Veterinária, graduada pela Universidade Presidente Antônio Carlos. Uberlândia-MG Brasil. \\ ${ }^{4}$ Médico Veterinário e Proprietário do Hospital Veterinário de Batatais. Batatais - SP Brasil. \\ ${ }^{5}$ Discente do curso de Medicina Veterinária na Universidade de Uberaba. Uberaba - MG Brasil. \\ ${ }^{6}$ Docente do curso de Medicina Veterinária na Universidade de Uberaba. Uberaba - MG Brasil. \\ *Autor para correspondência: E-mail: dilmamendes3m@yahoo.com.br
}

Resumo. O forame epiplóico é uma abertura natural na cavidade torácica que faz comunicação com a cavidade abdominal, localizado dorsalmente à fissura porta do fígado, fazendo contato com a veia cava caudal, veia porta, pâncreas, ligamento hepatoduodenal e com as alças do intestino delgado. Equinos diagnosticados com encarceramento de alça intestinal no forame epiplóico necessitam de tratamento cirúrgico, com prognóstico de reservado à desfavorável, sendo o íleo o segmento intestinal mais afetado nesta enfermidade. Além disso, por ocorrer um processo estrangulativo, o animal apresenta sinais de desconforto abdominal denominado de cólica e refluxo enterogástrico, porém os sinais clínicos são variáveis. Alguns animais na palpação apresentam intestino delgado palpável ou não e desconforto abdominal variando de leve a severo. O objetivo deste trabalho é relatar um caso de um equino da raça Quarto de Milha, fêmea, com 5 anos de idade, pesando $430 \mathrm{~kg}$, com histórico de desconforto abdominal há dois dias e com dores intermitentes, proveniente do município de Batatais, atendido no Hospital Veterinário de Batatais, abordando a clínica do animal, o diagnóstico e o tratamento. Na palpação retal identificou alças um pouco distendidas e intestino delgado palpável e a punção do líquido peritoneal através da abdominocentese mostrou-se com coloração avermelhada. $\mathrm{O}$ animal ficou em observação, porém mesmo com todas as intervenções clínicas, o quadro de dor aumentou e com isso foi submetido à laparotomia exploratória, onde se diagnosticou o encarceramento no forame epiplóico. Diante do caso clínico abordado, conclui-se que o encarceramento do forame epiplóico pode levar ao comprometimento de alças intestinais significativo, progredindo para desestabilização do estado geral do animal, sendo fatores agravantes para o procedimento cirúrgico, passando a ser um caso de risco para o paciente. $\mathrm{O}$ animal deste relato foi submetido a eutanásia devido ao grande comprometimento das alças intestinais. Conclui-se que o diagnóstico deve ser realizado o mais precoce possível aumentando a chance de resolução com prognóstico favorável.

Palavras chave: estrangulamento, cólica, cavalo

\section{Intestinal encarceration in epiplóico forame: Case report in equine}

Abstract. The epiplastic foramen is a natural opening in the thoracic cavity that communicates with the abdominal cavity, located dorsally to the cleft of the liver, contacting the caudal vena cava, portal vein, pancreas, hepatoduodenal ligament and with the small intestine loops. Horses diagnosed with intestinal loop incarceration in the epiplastic foramen require surgical treatment, with a prognosis of being reserved to unfavorable, and the ileum is the intestinal segment most affected in this disease. In 
addition, because of a strangulative process, the animal shows signs of abdominal discomfort called colic and enterogastric reflux, but clinical signs are variable. Some animals in the palpation present palpable small intestine or not and abdominal discomfort varying from mild to severe. The objective of this work is to report a case of a 5-year-old female Quarter Horse, weighing $430 \mathrm{~kg}$, with a history of abdominal discomfort two days ago and with intermittent pains, from the municipality of Batatais, Veterinary Hospital of Batatais, addressing the animal clinic, diagnosis and treatment. In the rectal palpation he identified slightly distended loops and palpable small intestine and puncture of the peritoneal fluid through the abdominocentesis showed a reddish coloration. The animal was under observation, but even with all the clinical interventions, the pain picture increased and with that it was submitted to exploratory laparotomy, where the incarceration was diagnosed in the epiplastic foramen. In view of the clinical case, it was concluded that the incarceration of the epiplastic foramen may lead to significant intestinal loops involvement, progressing to destabilization of the general state of the animal, being aggravating factors for the surgical procedure, becoming a case of risk of the patient. The animal in this report was submitted to euthanasia due to the great compromise of the intestinal loops. It is concluded that the diagnosis should be performed as early as possible, increasing the chance of resolution with favorable prognosis.

Keywords: strangulation, colic, horse

\section{Encarcelamiento intestinal en forame epiplóico: Relato de caso en equino}

Resumen. El foramen epiplóico es una abertura natural en la cavidad torácica que hace comunicación con la cavidad abdominal, localizada dorsalmente a la fisura de la puerta del hígado, haciendo contacto con la vena cava caudal, vena porta, páncreas, ligamento hepatoduodenal y también con las asas del intestino delgado. Los equinos diagnosticados con encarcelamiento de alza intestinal en el foramen epiplóico necesitan tratamiento quirúrgico, con pronóstico de reservado a la desfavorable, siendo el íleo el segmento intestinal más afectado en esta enfermedad. Además, por ocurrir un proceso estrangulativo, el animal presenta signos de incomodidad abdominal denominado cólico y reflujo enterogástrico, pero los signos clínicos son variables. Algunos animales en la palpación presentan intestino delgado palpable o no e incomodidad abdominal variando de leve a severo. El objetivo de este trabajo es relatar un caso de un yegua de la raza Cuarto de Milla, con edad de 5 años, pesando $430 \mathrm{~kg}$, con histórico de incomodidad abdominal hace dos días y con dolores intermitentes, proveniente del municipio de Batatais, atendido en el municipio de Batatais, Hospital Veterinario de Batatales, abordando la clínica del animal, el diagnóstico y el tratamiento. En la palpación rectal se identificaron tirantes un poco distendidas e intestino delgado palpable y la punción del líquido peritoneal a través de la abdominocentesis se mostró con coloración rojiza. El animal se quedó en observación, pero incluso con todas las intervenciones clínicas, el cuadro de dolor aumentó y con ello fue sometido a la laparotomía exploratoria, donde se diagnosticó el encarcelamiento en el foramen epiplóico. En el caso clínico abordado, se concluye que el encarcelamiento del foramen epiplóico puede llevar al compromiso de alzas intestinales significativo, progresando hacia la desestabilización del estado general del animal, siendo factores agravantes para el procedimiento quirúrgico, pasando a ser un caso de riesgo para sobrevida del paciente. El animal de este relato fue sometido a eutanasia debido al gran compromiso de las asas intestinales. Se concluye que el diagnóstico debe realizarse lo más precoz posible aumentando la probabilidad de resolución con pronóstico favorable.

Palabras clave: estrangulamiento, cólico, caballo

\section{Introdução}

A síndrome cólica é considerada uma emergência na medicina equina e uma das maiores causas de óbito nesta espécie (Southwood, 2013). Dentre as causas das cólicas, existem as estrangulativas e as não-estrangulativas (White, 1990). Dentre os tipos de cólicas, o encarceramento do forame epiplóico se 
encaixa nas causas estrangulativas (White, 1990). O forame epiplóico é chamado de forame de Winslow. É um orifício que permite a passagem de estruturas como veia porta, artéria hepática, ducto colédoco e veia cava caudal. Está localizada entre veia cava caudal e pâncreas (Auer \& Stick, 2012). As estruturas envolvidas nessa porção quando comprometidas podem culminar no bloqueio do lúmen intestinal e comprometimento circulatório do segmento encarcerado evoluindo para um possível quadro de estrangulamento (Southwood, 2013).

A hérnia de Winslow ou encarceramento está relacionado aos fatores como estresse, ansiedade e aerofagia, método de desmame e alimentação (Archer et al., 2004). A atrofia do bordo caudal do fígado pode levar ao afrouxamento do forame, predispondo a formação da hérnia causando o encarceramento neste espaço na cavidade abdominal (Auer \& Stick, 2012). Os sinais clínicos são variáveis e podem progredir para alterações sistêmicas (Freeman \& Pearn, 2015; Moore et al., 1976).

Para auxiliar no diagnóstico pode-se utilizar o ultrassom abdominal ou palpação retal (Freeman \& Pearn, 2015). A avaliação do líquido peritoneal serve como marcador para o processo estrangulativo, que dita ser um caso cirúrgico ou não. Nos casos de encarceramento no forame epiplóico, o tratamento é cirúrgico (Southwood, 2013). O prognóstico varia de reservado a desfavorável. O grau de estrangulamento e necessidade de ressecção de alça também influencia no prognóstico (Southwood, 2013).

O objetivo deste trabalho é relatar um caso de um equino atendido no Hospital de Equinos de Batatais diagnosticado com encarceramento no forame epiplóico abordando a clínica, o diagnóstico e o tratamento.

\section{Relato de caso}

Foi atendido no Hospital de Equinos de Batatais, um equino, de 5 anos de idade, da raça Quarto de Milha, fêmea, pesando $430 \mathrm{~kg}$. Foi encaminhado pelo veterinário responsável da propriedade, que relatou que o animal vinha sentindo desconforto abdominal há dois dias, porém as dores eram intermitentes. No dia anterior, um funcionário observou que o animal estava "rolando" na baia e o veterinário administrou dipirona sódica, seguido por cloridrato de tramadol e passou uma sonda nasogástrica, obtendo aproximadamente 20 litros de conteúdo espontâneo.

No Hospital Veterinário, ao realizar o exame físico, o animal apresentava-se com motilidade reduzida, mucosa normocorada, frequência cardíaca de 60 batimentos por minuto, frequência respiratória de 20 movimentos respiratórios por minuto, temperatura de $38,5^{\circ} \mathrm{C}$ e tugor cutâneo normal.

No momento do atendimento, o equino foi sedado com detomidina na dose de $0,02 \mathrm{mg} / \mathrm{kg} \mathrm{e} 1 \mathrm{mg} / \mathrm{kg}$ de N-butilbrometo de hioscina, ambos por via intravenosa, seguido por palpação retal na qual identificou-se alças pouco distendidas e intestino delgado palpável. Realizou-se tricotomia no ponto mais ventral da região da linha média do abdômen para punção do líquido peritoneal que se apresentou com coloração avermelhada (Figura 1).

As primeiras intervenções realizadas foram coleta de sangue (Figura 2) para exames de hemograma, bioquímico, hemogasometria e colocação do cateter central.

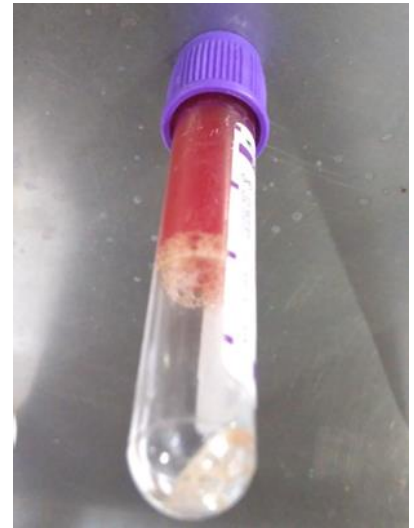

Figura 1. Líquido peritoneal coletado pela técnica de abdominocentese apresentando aspecto avermelhado, proteína de 5\% e hematócrito de $1 \%$.

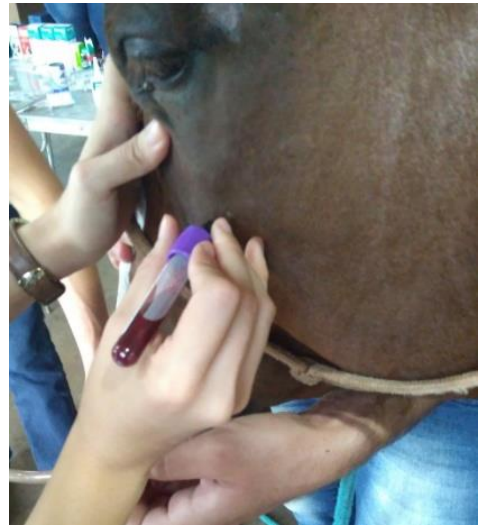

Figura 2. Coleta de sangue de um equino, da raça quarto de milha, de 5 anos de idade, atendido no Hospital Veterinário de Batatais-MG, realizado no dia 13 de fevereiro de 2018. 
$\mathrm{O}$ animal foi colocado na fluidoterapia com solução de ringer com lactato (aproximadamente 20 litros), sorbitol (200 ml diluído em 1 litro de ringer com lactato), cimetidina (dose: $4 \mathrm{mg} / \mathrm{kg}$, por via intravenosa), DMSO (dose: $1 \mathrm{~g} / \mathrm{kg}$ no volume de $400 \mathrm{ml}$ sendo $100 \mathrm{ml}$ para cada 1 litro de ringer com lactato), flunixina meglumina (dose: $1,1 \mathrm{mg} / \mathrm{kg}$ com volume total de $10 \mathrm{ml}$, por via intravenosa) e o paciente ficou em observação.

No exame de hemograma (Tabela 1), as alterações encontradas foram leucopenia, bastonete aumentado e trombocitopenia.

Tabela 1. Perfil hematológico de um equino, da raça quarto de milha, de 5 anos de idade, atendido no Hospital Veterinário de Batatais-MG, realizado no dia 13 de fevereiro de 2018

\begin{tabular}{lcc}
\hline Hemograma & Resultado & Referência \\
\hline Eritrograma & 8,30 milhões $/ \mathrm{mm}^{3}$ & $7,0-13$ \\
Hemácias & $12,1 \mathrm{~g} / \mathrm{dl}$ & $10-18$ \\
Hemoglobina & $37,6 \%$ & $32-55$ \\
Hematócrito & & \\
\hline Leucograma & 5,400 & $7-14$ \\
\hline Leucócitos & 30 & $0-2$ \\
Bastonete & 46 & $30-65$ \\
Segmentado & 0 & $0-2$ \\
Eosinófilo & 23 & $25-70$ \\
Linfócitos & 1 & $1-7$ \\
Monócito & 0 & $0-3$ \\
Basófilo & 127.000 & $200.000-600.000$ \\
Plaquetas & &
\end{tabular}

Fonte: Hospital Veterinário de Batatais

No exame bioquímico (Tabela 2), ureia, creatinina, ALT (Alanina aminotrasferase) e lactato se encontravam elevados e AST (Aspartato aminotransferase) abaixo dos valores de referência, seguido de hipoproteinemia.

Tabela 2. Perfil bioquímico de um equino, da raça quarto de milha, de 5 anos de idade, atendido no Hospital Veterinário de Batatais-MG, realizado no dia 13 de fevereiro de 2018.

\begin{tabular}{lcc}
\hline Bioquímica & Resultado & Referência \\
\hline Ureia & 66 & $21-54 \mathrm{mg} / \mathrm{dL}$ \\
Creatinina & 2,88 & $0,9-1,8 \mathrm{mg} / \mathrm{dL}$ \\
Proteína total & 66 & $56-80 \mathrm{~g} / \mathrm{l}$ \\
AST & 22 & $50-150 \mathrm{U} / \mathrm{mL}$ \\
ALT & 31 & $7-20 \mathrm{U} / \mathrm{mL}$ \\
GGT & 13 & $1,3-13 \mathrm{U} / \mathrm{L}$ \\
Lactato & 15,7 & $1,11-1,78 \mathrm{Mmol} / \mathrm{L}$
\end{tabular}

Fonte: Hospital Veterinário de Batatais

Na hemogasometria (Tabela 3), $\mathrm{PO}_{2}, \mathrm{~K}+, \mathrm{CL}^{-}$e $\mathrm{BE}$ apresentaram-se abaixo dos valores de referência. Após 6 horas, o animal apresentou aumento do quadro de dor com inquietação, desconforto e mucosa oral com formação de halo endotoxêmico, sendo necessária a intervenção cirúrgica, pois não se tratava de um caso de resolução clínica.

$\mathrm{O}$ animal foi direcionado para sala de indução onde foi administrado $0,05 \mathrm{mg} / \mathrm{kg}$ de detomidina para sedação, $2 \mathrm{mg} / \mathrm{kg}$ de cetamina e $250 \mu \mathrm{g} / \mathrm{ml}$ de dobutamina para indução anestésica. O paciente foi posicionado em decúbito dorsal na mesa cirúrgica e mantido na anestesia inalatória com isoflurano 0,5\%. Preparou-se a área de campo cirúrgico, com tricotomia do abdômen, antissepsia com iodopolvidona, degermante tópico e álcool $70 \%$ e foi realizada a celiotomia exploratória, com acesso pré-umbilical pela linha média. 
Tabela 3. Exame de hemogasometria de um equino, da raça quarto de milha, de 5 anos de idade, atendido no Hospital Veterinário de Batatais- MG, realizado no dia 13 de fevereiro de 2018.

\begin{tabular}{lcc}
\hline Hemogasometia & Resultado & Referência \\
\hline $\mathrm{pH}$ & 7,38 & $7,35-7,45$ \\
$\mathrm{pCO}_{2}$ & $41,8 \mathrm{mmHg}$ & $36-44 \mathrm{mmHg}$ \\
$\mathrm{PO}_{2}$ & $28,9 \mathrm{mmHg}$ & $61,3-100 \mathrm{mmHg}$ \\
$\mathrm{K}+$ & $3,25 \mathrm{mmo} / \mathrm{L}$ & $3,5-5,10 \mathrm{mmo} / \mathrm{L}$ \\
$\mathrm{CL}^{-}$ & $94 \mathrm{mmo} / \mathrm{L}$ & $96-108 \mathrm{mmo} / \mathrm{L}$ \\
$\mathrm{Hct}$ & $49 \%$ & $34-52 \%$ \\
$\mathrm{BE}$ & $-0,6$ & $-3,5 \mathrm{a}+3,5 \mathrm{mEq} / \mathrm{L}$ \\
\hline
\end{tabular}

Fonte: Hospital Veterinário de Batatais

Durante a inspeção, observou-se que parte do intestino delgado estava comprometida, com aproximadamente 6 metros de alça desvitalizada e necrosada (Figura 3 A, B e C). Após manipulação trans-operatória conseguiu-se realizar o reposicionamento da alça, porém foi observado que o quadro do animal era desfavorável e de difícil resolução no pós-cirúrgico.
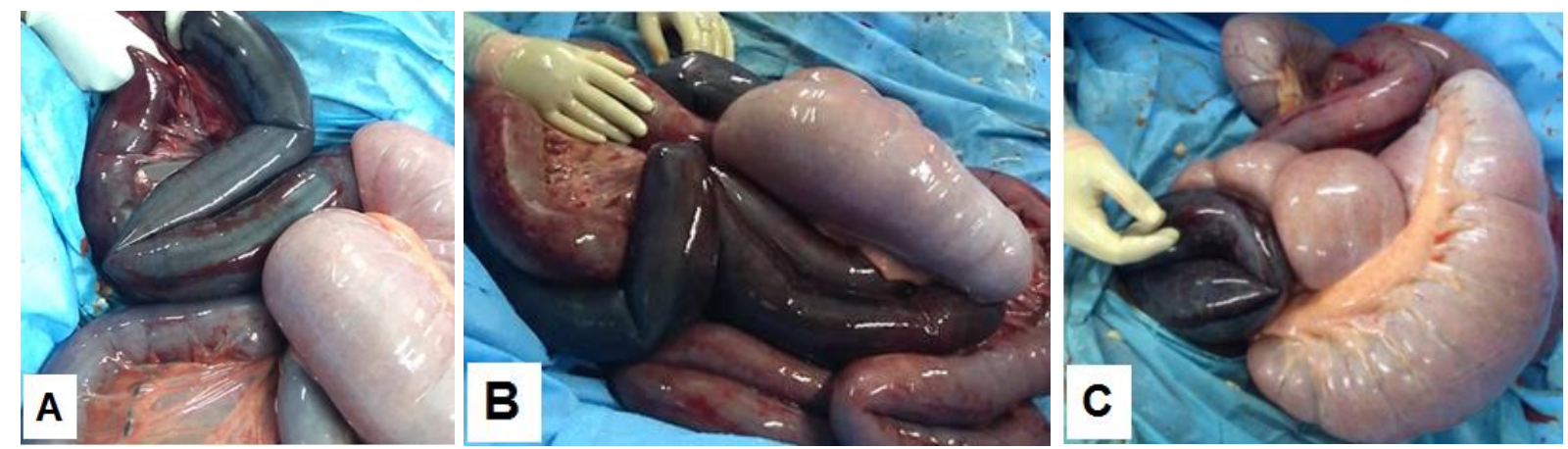

Figura 3 A, B e C: Laparotomia exploratória realizada no dia 13 de fevereiro de 2018 no Hospital Veterinário de Equinos de Batatais, onde aproximadamente 6 metros de alças do intestino delgado estava encarcerada no forame epiplóico, com perda de vitalidade e necrosadas.

A partir do parecer do proprietário e da condição que se encontrava as alças, optou-se pela eutanásia, onde foi administrado 21 ampolas de cloreto de potássio por via intravenosa.

\section{Discussão}

Segundo Archer et al. (2008), equinos acometidos por encarceramento de forame epiplóico nem sempre apresentam sinais típicos de cólica estrangulativa de intestino delgado, além de não apresentar na palpação retal intestino delgado palpável, porém, no relato de caso citado, o animal apresentou sinais clínicos semelhantes aos de cólica estrangulativa, com quadro de dor moderada a severa e refluxo gástrico, ressaltando que na palpação, o equino deste caso apresentava intestino delgado palpável e pouco distendido.

Freeman \& Pearn (2015) afirmam que os sinais clínicos podem ser variáveis, devido a ter relação com outras afecções como enterite proximal ou até mesmo lipoma, e neste caso, o refluxo gástrico pode estar presente ou não. Para auxiliar no diagnóstico realiza-se a palpação retal e ressalta-se que as alças intestinais nem sempre apresentam distendidas, não sendo um diagnóstico confirmatório, onde se deve complementar com outros exames como ultrassom abdominal, que é útil para diagnóstico de processos estrangulativos. Nos exames clínicos realizados no caso relatado ocorreu refluxo gástrico, alças intestinais distendidas á palpação e não se realizou ultrassom abdominal.

Nos exames laboratoriais, observou-se que o animal apresentava uma leucopenia e bastonetes aumentados. Faleiros et al. (2001) afirmam que essas alterações são contundentes durante a evolução da síndrome cólica, em razão das células de defesa migrar para as alças intestinais comprometidas ou para a cavidade abdominal em casos de peritonite, o que se assemelhou com o animal do caso relatado. Segundo Johnson (1995) e Seahorn \& Seahorn (2003), a hipocalemia em equinos com cólica é um 
achado frequente, pois ocorre devido a fatores como alcalose e hiperinsulinemia, como o caso citado, na qual a hipocalemia esteve presente, porém o animal não apresentou alcalose e a hiperinsulinemia não foi observada, pois a insulina não foi dosada.

O cloro é secretado no estômago e absorvido pela porção final do intestino delgado e distúrbios, como refluxo gástrico, compromete a sua absorção, como afirmam Seahorn \& Seahorn (2003). White (1990) também ressalta que a hipocloremia pode ocorrer por sequestro gastrintestinal e repetidas lavagens gástricas, o que também foi apresentado no animal relatado, apresentando hipocloremia na hemogasometria.

De acordo com Magdesian (2004), o nível de lactato é utilizado como prognóstico em equinos com cólica, onde constatou que em concentrações superiores a $11.2 \mathrm{mEq} / \mathrm{L}$ o prognóstico é pior que em paciente com $8.3 \mathrm{mEq} / \mathrm{L}$. Todavia, Moore et al. (1976) dita que os pacientes com concentração superiores a $8 \mathrm{mmol} / \mathrm{L}$ já tem um prognóstico desfavorável. Neste caso, a concentração de lactato se encontrava superior aos valores citados pelos autores, com 15,7 mmol/L, tornando-se desfavorável.

As concentrações de ureia e creatinina são importantes para o animal em processo de desidratação e para avaliar a função renal. Equino com cólica abdominal é susceptível a apresentar azotemia pré-renal, tornando um caso de grave a severo, podendo até mesmo levar a falência renal aguda (Radostits et al., 2010). Di Filippo et al. (2009) afirmam que as concentrações aumentadas desses biomarcadores de função renal estão relacionadas com a hipotensão, septicemia e com a coagulação intravascular disseminada. No presente trabalho, observou-se que o paciente apresentava os valores de ureia e creatinina aumentados, porém, o mesmo não demorou a ser direcionado para o procedimento cirúrgico e posteriormente ser eutanasiado.

O líquido obtido durante a paracentese quando possui alta celularidade, possui aspecto turvo e sanguinolento, indicando que há desvitalização de alças intestinais, com indicação cirúrgica, de acordo com White (1990) e Archer et al. (2008). Neste relato de caso, após coleta de líquido peritoneal, com aspecto sanguinolento, o animal ficou em observação por seis horas e não foi encaminhado de imediato para intervenção cirúrgica.

O animal relatado tinha cinco anos de idade. Segundo Southwood (2013), o encarceramento do forame epiplóico acomete animais acima de seis anos de idade, o que não condiz com o relato de caso. Auer \& Stick (2012) afirmam que cavalos de qualquer faixa etária podem ser afetados, cavalos de 11 meses a três anos sendo mais raro em equinos com mais de 20 anos de idade. Animais com idade superior a seis anos têm sido apontados como mais predispostos a herniação neste forame, devido à atrofia no lobo caudal direito do fígado, o que aumentaria o espaço e a probabilidade de uma alça de intestino delgado se insinuar neste forame (Southwood, 2013). Todavia, Auer \& Stick (2012) apontam que a idade do animal não tem relação com a atrofia do fígado e afrouxamento do forame epiplóico e consequentemente levar a causa do encarceramento.

Segundo Smith et al. (2009) e Zachary et al. (2012), o encarceramento no forame epiplóico pode comprometer a circulação arteriovenosa, isquemia, infarto hemorrágico e pode levar a um choque hipovolêmico, prosseguindo para um quadro irreversível e morte do animal. No relato de caso, foi observado, mucosa com halo cianótico característico de endotoxemia, porém, o animal não veio a óbito, e optou-se pela eutanásia durante o transoperatório em razão do pós-operatório ser delicado e de difícil reversão pela extensão da lesão. Estudos realizados por Auer \& Stick (2012), entre as cirurgias de cólica, verificaram que o encarceramento do forame epiplóico foi a única variável associada a diminuição da sobrevida. Já Cook (2011) afirma que os prognósticos são menores em equinos com encarceramento no forame epiplóico, com mortalidade resultando em uma taxa de sobrevivência de 55\% no período de um ano, porém não se sabe a razão da diferença, mas que é um dado importante para o proprietário. Segundo Southwood (2013), o tempo de cirurgia também influencia no prognóstico, sendo favorável no póscirúrgico de $95 \%$ em curto prazo e desfavorável com $70 \%$ em longo prazo, correlacionando com caso relatado.

\section{Conclusão}

O encarceramento do forame epiplóico é uma afecção de grande importância dentro das causas de abdômen agudo diagnosticadas em laparotomia exploratória em equinos. Devido ao fato de ser uma 
causa classificada como estrangulante, compromete a sobrevida do animal, sendo de extrema importância um diagnóstico precoce e encaminhamento pelo médico veterinário.

\section{Referências bibliográficas}

Archer, D. C., Pinchbeck, G. L., French, N. P. \& Proudman, C. J. (2008). Risk factors for epiploic foramen entrapment colic: an international study. Equine Veterinary Journal, 40(3):224-230.

Archer, D. C., Proudman, C. J., Pinchbeck, G., Smith, J. E., French, N. P. \& Edwards, G. B. (2004). Entrapment of the small intestine in the epiploic foramen in horses: a retrospective analysis of 71 cases recorded between 1991 and 2001. Veterinary Record, 155(25):793-797.

Auer, J. A. \& Stick, J. A. (2012). Equine Surgery. USA: Elsevier Health Science.

Cook, V. L. 2011. Surgical Options and Prognosis for the Acute Abdomen: Small Intestine. 5 f. Tese (Doutorado) - Curso de Veterinary Medicine, Department Of Large Animal Clinical Sciences, College of Veterinary Medicine, Indianapolis, In, USA.

Di Filippo, P. A., Santana, A. E. \& Coleta, F. E. D. (2009). Avaliação clínica e eritroleucograma de eqüinos com cólica submetidos a laparotomia, sobreviventes e não sobreviventes. Ciência Animal Brasileira, 10(4):1246-1255.

Faleiros, R. R., Alves, G. E. S., Santos, R. L., Marques Júnior, A. P. \& Macoris, D. G. (2001). Experimental ischemia and reperfusion in equine small colon. Arquivo Brasileiro de Medicina Veterinária e Zootecnia, 53(3):341-350.

Freeman, D. E. \& Pearn, A. R. (2015). Anatomy of the vestibule of the omental bursa and epiploic foramen in the horse. Equine Veterinary Journal, 47(1):83-90.

Johnson, P. J. (1995). Electrolyte and acid-base disturbances in the horse. Veterinary Clinics of North America: Equine Practice, 11(3):491-514.

Magdesian, K. G. (2004). Monitoring the critically ill equine patient. The Veterinary clinics of North America. Equine practice, 20(1):11-39.

Moore, J. N., Owen, R. R. \& Lumsden, J. H. (1976). Clinical evaluation of blood lactate levels in equine colic. Equine Veterinary Journal, 8(2):49-54.

Radostits, O. M., Gay, C. C., Blood, D. C., Hinchcliff, K. W. \& McKenzie, R. A. (2010). Clínica Veterinária: um tratado de doenças dos bovinos, ovinos, suínos, caprinos e eqüinos (Vol. 1). Rio de Janeiro: Guanabara Koogan.

Seahorn, J. L. \& Seahorn, T. L. (2003). Fluid therapy in horses with gastrointestinal disease. The Veterinary Clinics of North America. Equine practice, 19(3):665-679.

Smith, M. A., Levine, D. G., Getman, L. M., Parente, E. J. \& Engiles, J. B. (2009). Vulvar squamous cell carcinoma in situ within viral papillomas in an aged Quarter Horse mare. Equine Veterinary Education, 21(1):11-16.

Southwood, L. L. (2013). Practical guide to equine colic: John Wiley \& Sons, Inc., Publication.

White, N. A. (1990). Epidemiology and etiology of colic. In N. A. White (Ed.), The equine acute abdomen (pp. 50-64). Philadelphia, USA: Lea and Febiger.

Zachary, J. F., McGavin, D. \& McGavin, M. D. (2012). Bases da patologia em veterinária. Rio de Janeiro: Elsevier Brasil.

Recebido: 19 de abril, 2019.

Aprovado: 15 de maio, 2019

Publicado: 4 de junho, 2019.

Licenciamento: Este artigo é publicado na modalidade Acesso Aberto sob a licença Creative Commons Atribuição 4.0 (CC-BY 4.0), a qual permite uso irrestrito, distribuição, reprodução em qualquer meio, desde que o autor e a fonte sejam devidamente creditado. 